\author{
M. HAHN ${ }^{1, \infty}$ \\ O. BARBIERI ${ }^{1}$ \\ F.P. CAMPANA ${ }^{1}$ \\ R. KÖTZ ${ }^{1}$ \\ R. GALLAY ${ }^{2}$
}

\title{
Carbon based double layer capacitors with aprotic electrolyte solutions: the possible role of intercalation/insertion processes
}

\author{
${ }^{1}$ Electrochemistry Laboratory, Paul Scherrer Institute, 5232 Villigen PSI, Switzerland \\ ${ }^{2}$ Maxwell Technologies SA, 1728 Rossens, Switzerland
}

Received: 5 October 2004/Accepted 17 August 2005

Published online: 18 November 2005 • C Springer-Verlag 2005

ABSTRACT The extraordinary stability and cycle life performance of today's electrochemical double-layer capacitors (EDLCs) are generally ascribed to the fact that charge storage in activated carbon (AC) is based on pure double-layer charging. In contrast, Faradaic charge-transfer reactions like those occurring in batteries are often connected with dimensional changes, which can affect the cycle life of these storage devices. Here we report the charge-induced height change of an AC electrode in an aprotic electrolyte solution, $1 \mathrm{~mol} / 1\left(\mathrm{C}_{2} \mathrm{H}_{5}\right)_{4} \mathrm{NBF}_{4}$ $\left(\mathrm{TEABF}_{4}\right)$ in acetonitrile. The results are compared with those obtained for a graphite electrode in the same electrolyte. For both electrodes, we observe an expansion/contraction of several percent for a potential window of $\pm 2 \mathrm{~V}$ vs. the immersion potential (ip). For the EDLC electrode, significant expansion starts at about $1 \mathrm{~V}$ remote from the ip and hence is well within the normal EDLC operation range. For the graphite electrode, the height changes are unambiguously caused by intercalation/deintercalation of both anions and cations. The close analogies between the graphite and the EDLC electrode suggest that ion intercalation or insertion processes might play a major role for charge storage, self discharge, cyclability, and the voltage limitation of EDLCs.

PACS 82.47.Uv; 82.45.Fk; 82.45.Gj; 82.80.Fk; 81.05.Uw

1

\section{Introduction}

The much higher stability and cycle life performance of today's electrochemical double-layer capacitors (EDLCs) when compared to batteries are generally ascribed to the circumstance that charge storage is based on pure double-layer charging, and not on Faradaic reactions. Such charge-transfer processes are often accompanied by microscopic structural changes, which may be not fully reversible and thus limit the cycle life of batteries.

In general, microscopic structural changes will result in macroscopic dimensional changes of the electrode. Consequently, dilatometry is a convenient means to distinguish between Faradaic (chemical) and capacitive (electrostatic) processes. This is especially true for poorly crystalline materials

Fax: +41 56310 4415, E-mail: matthias.hahn@psi.ch like activated carbon (AC), where diffraction experiments often give no satisfactory information.

For carbon materials, several possible phenomena have been identified which may lead to charge-induced dimensional changes: (i) an increase of the interlayer distance upon ion intercalation between adjacent basal planes $[1,2]$, (ii) widening of the intralayer $\mathrm{C}-\mathrm{C}$ bond length upon electron injection into the aromatic planes (and shrinking upon hole injection) [3-8], and (iii) an expansion due to the decrease of surface tension with increasing excess charge in the electrochemical double layer [9-11].

For graphite, intercalation of a large variety of ions of either sign is well established. The accompanying $c$-axisdistance widening ranges from about $10 \%$ for $\mathrm{Li}^{+}$(in the compound $\mathrm{LiC}_{6}$ ) [2] to more then hundred percent for some large ions [1].

Besides this large $c$-axis expansion a much smaller effect (about $1.5 \%$ at maximum) of intercalation was predicted [35] and measured [5-8] for the intralayer $\mathrm{C}-\mathrm{C}$ bond length. The corresponding strain, being due to quantum-mechanical effects (changes in orbital occupation and band structure), changes sign from an expansion for electron injection to a contraction for hole injection. Notably, this effect is expected to occur for double-layer charging as well.

The third postulated effect is based on the decrease of surface tension with increasing excess charge in the electrochemical double layer. This effect, well established for the liquid-mercury electrode, would lead to an expansion of a solid spherical electrode upon charging in either direction and consequently also to an expansion of a porous electrode composed of such spheres. In fact, the predicted linear relation between strain and excess charge of either sign remote from the potential of zero charge (pzc) has been observed for different carbon electrodes in neutral aqueous solutions [9-11]. However, the reported overall height change is very small, below $0.05 \%$.

In the present contribution we report measurements of dimensional changes of an AC electrode used for electrochemical double layer capacitors in organic, aprotic electrolyte solutions, where higher charge densities can be obtained than with neutral aqueous solutions. The results obtained are compared with corresponding experiments performed with a graphite electrode, and are discussed on the basis of the above mechanisms. 
2

\section{Experimental}

The electrochemical measurements were performed with a purpose-made dilatometer (Fig. 1). Related set-ups were used by Biberacher et al. [12], Besenhard et al. [13], Winter et al. [14], and Ohzuku et al. [15]. Basically the dilatometer consists of a cell stack, i.e. two electrodes separated by a paper separator. The lower electrode is fixed and serves as the counter electrode (CE) while the upper electrode serves as the working electrode (WE) and is free to move against a constant load $(20 \mathrm{~N})$ applied by means of a spring. The overall height change of the cell is monitored by an inductive displacement transducer (W5TK, in combination with a measuring amplifier MGT 233.D4, both by Hottinger Baldwin Messtechnik, Germany) mounted on top of the plunger that contacts the WE. In order to avoid any pressure build up due to gas evolution, the cell was connected to atmospheric pressure via a drying agent (molecular sieve $0.4 \mathrm{~nm}$, Merck) filled column.

A small stripe of the EDLC electrode material $\left(2 \times 4 \mathrm{~mm}^{2}\right)$ being fixed and contacted by an aluminum wire served as the reference electrode. All electrode potentials are referred to the immersion potential (ip) of the EDLC electrode, $3.05 \mathrm{~V}$ vs. $\mathrm{Li} / \mathrm{Li}^{+}$. The potential was controlled by means of a potentio-

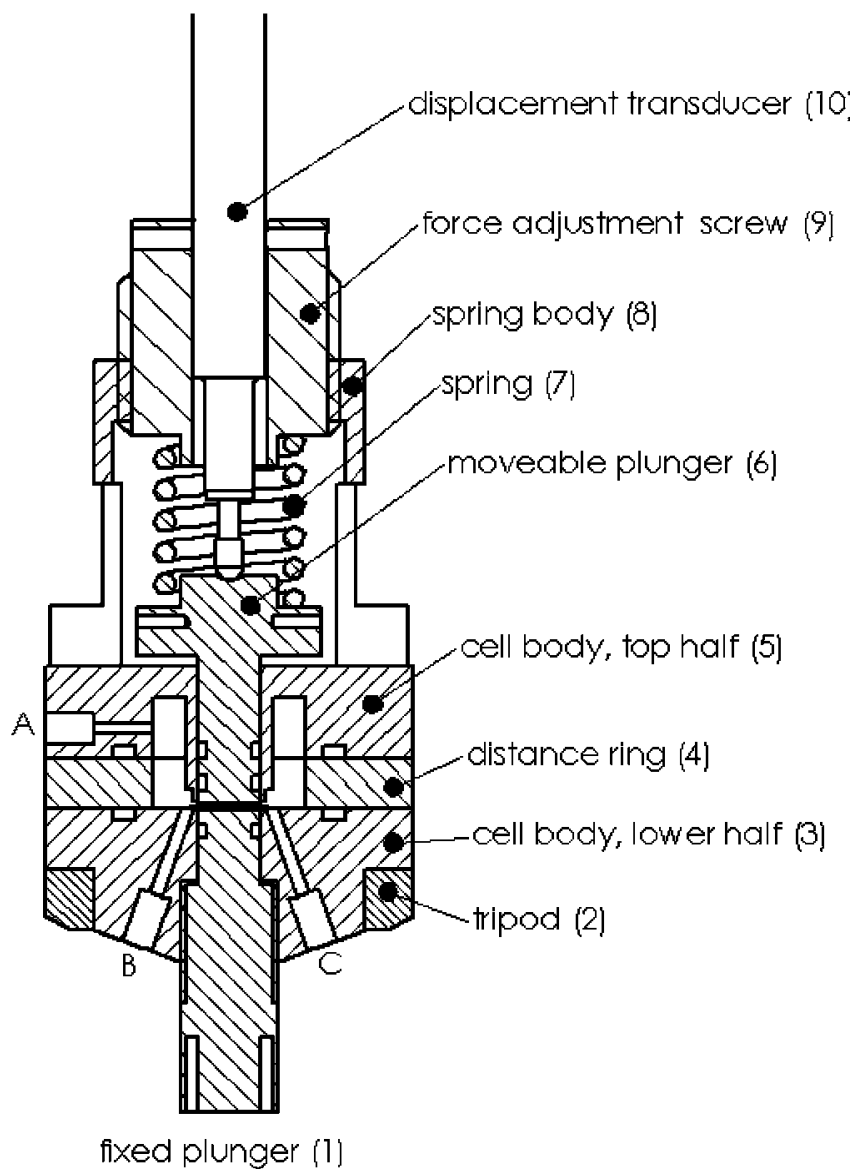

FIGURE 1 Dilatometer in the full cell configuration. The cell stack is located between the two plungers (1 and 6). One opening (B) serves as the electrolyte solution inlet. The second opening (A) connects the cell volume via a drying column to atmospheric pressure. An aluminum wire contacting the reference electrode is introduced through the third opening $(\mathbf{C})$ stat (model 273A, EG\&G Princeton Applied Research). Some electrochemical impedance measurements were performed in combination with an impedance/gain-phase analyzer (model 1260, Solartron).

The cell components are made of titanium grade 2 (current collectors), PEEK (housing), and EPDM (sealings). A commercial EDLC electrode material was employed, which consists of a 0.15-mm-thick PTFE-bound activated carbon film. One series of measurements was performed with a graphite electrode. This electrode was prepared by spin coating an aqueous suspension of graphite (KS44, Timcal, with $10 \mathrm{wt}$ \% carboxymethylcellulose sodium salt, Merck) on an etched aluminum foil (0.03-mm thick) and subsequent drying. By repeating this procedure several times a $0.016-\mathrm{mm}$-thick coating with a total mass of $1.2 \mathrm{mg}$ was prepared.

After assembly in air the dilatometer (with the displacement transducer detached) was dried for $24 \mathrm{~h}$ at $140{ }^{\circ} \mathrm{C}$ and $<0.01$ mbar in a vacuum oven, then cooled to ambient temperature in argon atmosphere, transferred to a glove box (argon, $<10 \mathrm{ppm}$ water), and finally filled there with the electrolyte solution, $1 \mathrm{~mol} / 1$ tetraethylammoniumtetrafluoroborate $\left(\mathrm{TEABF}_{4}\right)$ in acetonitrile $(<20 \mathrm{ppm}$ water). Measurements were performed outside the glove box in a test cabinet at constant temperature $\left(25 \pm 0.05^{\circ} \mathrm{C}\right)$.

The dilatometer was used in three different configurations.

(i) For measurements of EDLC full cell height changes two identical electrodes (10-mm diameter, 0.15-mm thick, $8.5 \mathrm{mg}$ ) were used, and the cell voltage was controlled. The full cell equivalent series resistance (real part of the impedance) measured at $0 \mathrm{~V}$ was about $0.8 \Omega$ at $1 \mathrm{kHz}$ and $2 \Omega$ at $0.01 \mathrm{~Hz}$.

(ii) For EDLC half cell measurements the WE (10-mm diameter, 0.15-mm thick) was combined with a large $\mathrm{CE}$ (diameter $18 \mathrm{~mm}$, height $2 \mathrm{~mm}$ ) made from the same EDLC electrode material. Due to the much higher capacitance, the $\mathrm{CE}$ potential stays almost pinned. The WE potential was controlled with respect to the reference electrode. In this configuration the full cell equivalent series resistance was about $1.3 \Omega$ at $1 \mathrm{kHz}$ and $7 \Omega$ at $0.01 \mathrm{~Hz}$.

(iii) Half cell measurements of the graphite electrode were performed using a large CE (18-mm diameter, 2-mm thick) made from the same EDLC electrode material. The potential of the much smaller graphite electrode $(10-\mathrm{mm}$ diameter, $0.016-\mathrm{mm}$ thick) was controlled with respect to the reference electrode.

The dilatometer was designed to minimize parasitic background currents stemming from other components than the carbon electrodes themselves. However, at extreme positive potentials, a small parasitic background current was caused by the titanium current collectors. The reported cyclic voltammograms and Coulombic efficiencies are not corrected for this background.

The resolution of the dilatometer was about $20 \mathrm{~nm}$. In the discharged state $(U=0)$ and after a settling period of $6 \mathrm{~h}$ (after filling) drifts of $<100 \mathrm{~nm} / \mathrm{h}$ and $<300 \mathrm{~nm} /$ day were measured.

A nitrogen adsorption measurement of the EDLC electrode material was performed with an ASAP 2010 apparatus (Micromeritics) at $77 \mathrm{~K}$. Prior to measurement the sample was 
outgassed at $250^{\circ} \mathrm{C}$ for $24 \mathrm{~h}$. From the isotherm the pore-size distribution was calculated according to the density functional theory (DFT) method [16] assuming slit-shaped pores.

\section{$3 \quad$ Results \\ 3.1 Pore-size distribution}

The pore-size distribution as obtained by DFT analysis of the nitrogen adsorption isotherm is shown in Fig. 2. For the incremental surface area two maxima are observed around $0.7-\mathrm{nm}$ and $1.3-\mathrm{nm}$ pore widths. The cumulative surface dred plot shows that almost the entire surface is generated by pores below $2 \mathrm{~nm}$.

\section{2}

\section{Voltammetry and height change}

Several cycles of the cyclic voltammogram (CV) and the concomitant height changes of an EDLC cell (configuration (i), see Sect. 2) are shown in Fig. 3. The current is normalized to the weight of the dry electrodes and the sweep

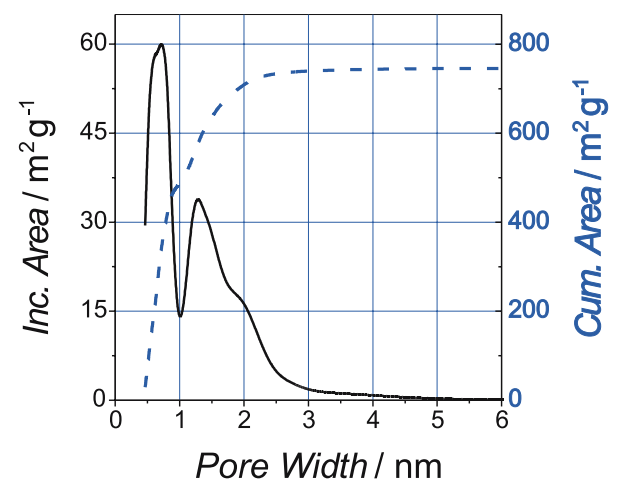

FIGURE 2 Incremental surface area (solid line) and cumulative surface area (dashed line) of the EDLC electrode material as obtained by DFT analysis of the nitrogen adsorption isotherm

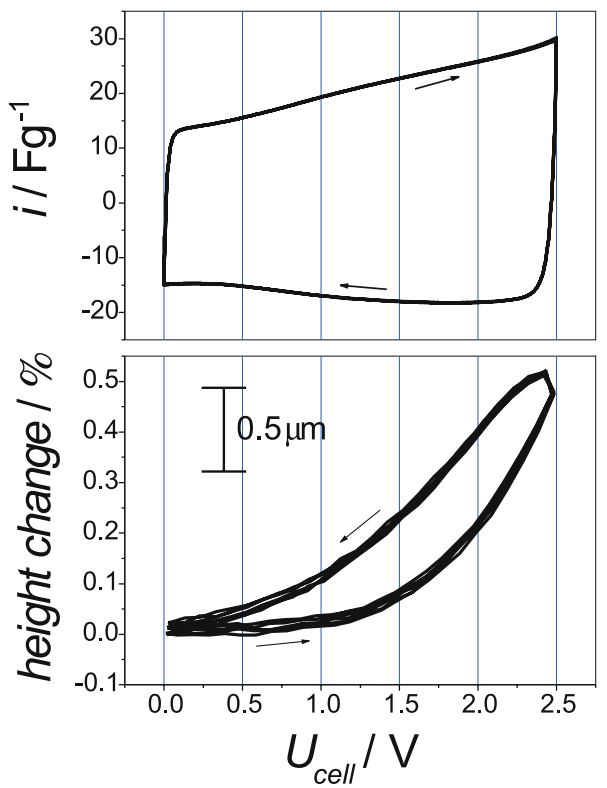

FIGURE $3 \mathrm{CV}$ (second to fifth cycles, top) and simultaneous dilatation record of a full EDLC cell with a total electrode height of $0.3 \mathrm{~mm}$. Scan rate: $20 \mathrm{mV} / \mathrm{s}$. Electrolyte solution: $1 \mathrm{M} \mathrm{TEABF}_{4}$ in acetonitrile rate, thus yielding the gravimetric cell capacitance. The CV shows almost ideal capacitor behavior with a slight increase of the capacitance towards the charged state. During charging, we observe a smooth increase of the cell height with perpetually increasing slope. A significant expansion of the cell is evident for voltages above $1.5 \mathrm{~V}$. A distinct hysteresis between the charging and the discharging branches can be seen. At the upper vertex points of the CV $(2.5 \mathrm{~V})$, i.e. for the fully charged capacitor cell, half cell potentials of $-1.35 \mathrm{~V}$ and $+1.15 \mathrm{~V}$, respectively, were measured.

In order to estimate the time response of the electrode expansion we performed CVs with different scan rates. Both the overall height change (amplitude) and the integral capacitance decrease with increasing scan rate (at $U=2.5 \mathrm{~V}$, Fig. 4a). From the time response of the height change when applying a staircase voltage profile ( 0 to $2.5 \mathrm{~V}$ ) one can determine a time constant of only a few seconds for the expansion process (Fig. 4b).

In order to distinguish between the contributions of the two electrodes, we performed half cell measurements in the respective potential ranges of the single electrodes $(0$ to $-1.35 \mathrm{~V}$ for the negative, and 0 to $+1.15 \mathrm{~V}$ for the positive). For this purpose we used the dilatometer with a large $\mathrm{CE}$ (configuration (ii)). In this set-up the CE potential stays almost constant and, since we expect no dimensional changes close to the ip, any observed changes can be attributed to the WE. The results obtained with one and the same electrode, first for cycling in the negative, and then for cycling in the positive potential range, are shown in Fig. 5. For the positive as well as for the negative $\mathrm{CV}$ dimensional changes of the electrode are evidenced with a maximum expansion of $0.25 \mu \mathrm{m}$ for the pos-
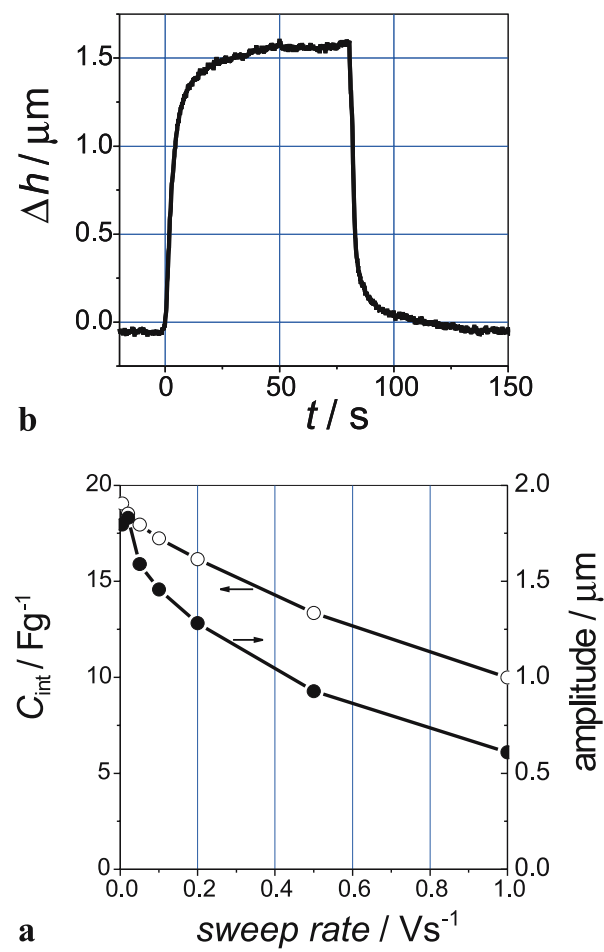

FIGURE 4 (a) Integral capacity and full cell height change (amplitude) upon cycling between 0 and $2.5 \mathrm{~V}$ as a function of the scan rate. (b) Time response when applying a staircase voltage profile ( 0 to $2.5 \mathrm{~V}$ ) 


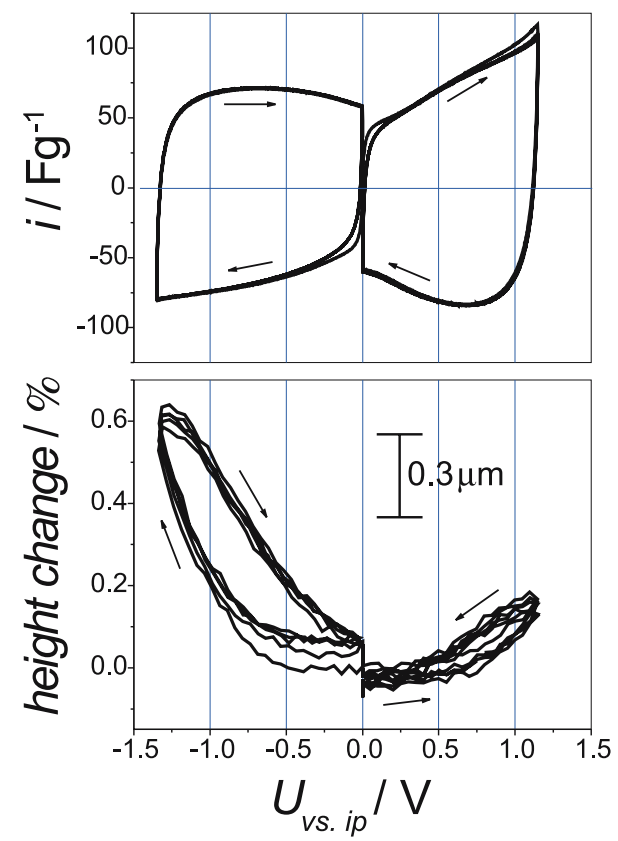

FIGURE 5 Top: EDLC half cell CVs (first to fifth cycles) for the negative $(0$ to $-1.35 \mathrm{~V})$ and the positive $(0$ to $1.15 \mathrm{~V})$ potential ranges. Scan rate: $10 \mathrm{mV} / \mathrm{s}$. Bottom: simultaneous dilatation record

itive and $1.0 \mu \mathrm{m}$ for the negative $\mathrm{CV}$. The sum of both values is in reasonable agreement with the value of $1.4 \mu \mathrm{m}$ obtained for the full cell with two electrodes in Fig. 3. Thus, for a capacitor voltage of $U=2.5 \mathrm{~V}, 75 \%$ of the full cell expansion (and most of the hysteresis) occurs at the negative electrode. Maximum charge capacities of $-86.3 \mathrm{~A} \mathrm{~s} / \mathrm{g}$ (with $99.5 \%$ Coulombic efficiency) and $83.0 \mathrm{~A} \mathrm{~s} / \mathrm{g}$ (with $98.0 \%$ Coulombic efficiency) were determined for the third cycle of the negative and the positive voltage ranges, respectively.

The situation changes when extending the voltage window (Fig. 6). For the negative potential range ( 0 to $-2 \mathrm{~V}$ ) a much more pronounced height change of several $\mu \mathrm{m}$ is now observed. On the other hand, the CV retains its featureless shape and quasi-stationary behavior is again attained within a few cycles. The maximum charge capacity is $-149.5 \mathrm{~A} \mathrm{~s} / \mathrm{g}$ with 98.7\% efficiency (third cycle).

For the extended positive range $(0$ to $+2 \mathrm{~V})$ the height change is again smaller than for the negative range but no longer fully reversible, and a continuous swelling of the electrode is observed during cycling. At the same time, a Coulombic efficiency of only $75.5 \%$ (third cycle) and a maximum charge capacity of $237.6 \mathrm{~A} \mathrm{~s} / \mathrm{g}$ are found. In the potential range between $1.5 \mathrm{~V}$ and $2.0 \mathrm{~V}$ a current increase is observed during the forward scan, which has no counterpart in the subsequent backward scan. Measurements with the sample-free dilatometer indicate that the poor Coulombic efficiency is not mainly due to parasitic charge transfer at the titanium current collector.

To cross check and to quantify our intercalation/insertion hypothesis, we also performed measurements with a graphite electrode (dilatometer in the half cell configuration (iii)). The results obtained with one and the same graphite electrode, first for cycling in the negative potential range $(0$ to $-2.0 \mathrm{~V}$ vs. the EDLC immersion potential), and then for cycling in the positive potential range $(0$ to $+2.5 \mathrm{~V})$, are shown in
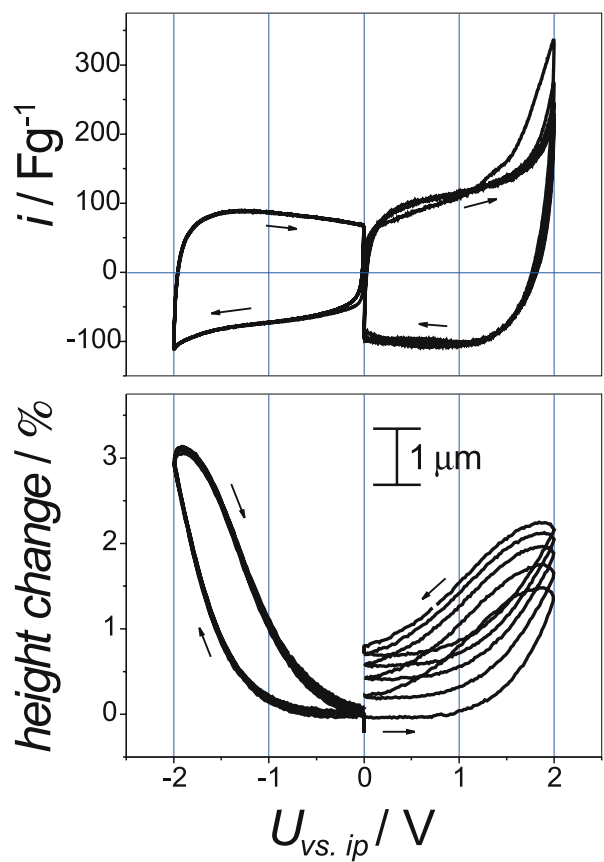

FIGURE 6 Top: EDLC half cell CVs (first to fifth cycles) for the extended negative $(0$ to $-2 \mathrm{~V})$ and the extended positive $(0$ to $2 \mathrm{~V})$ potential ranges. Scan rate: $10 \mathrm{mV} / \mathrm{s}$. Bottom: simultaneous dilatation record

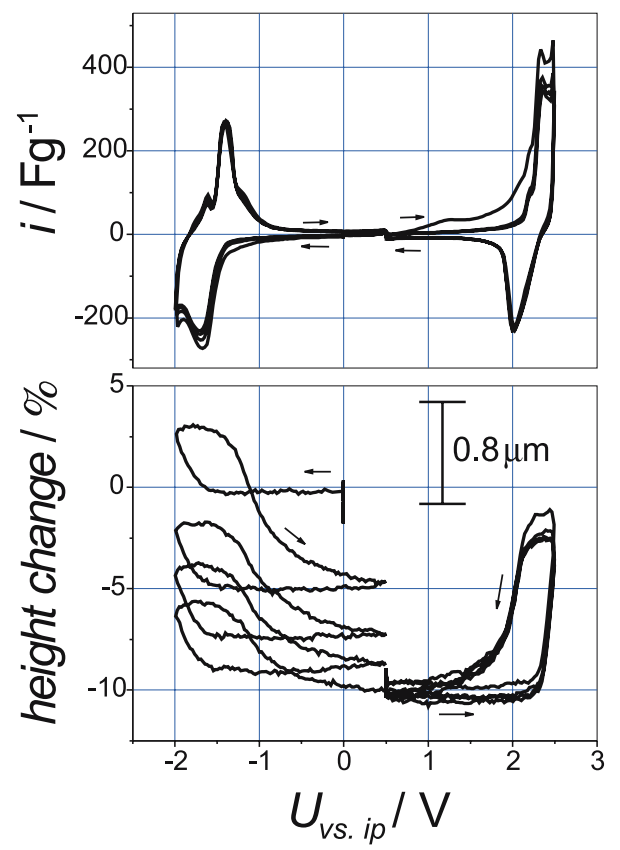

FIGURE 7 Top: graphite half cell CVs (first to fourth cycles) for the negative $(0$ to $-2.0 \mathrm{~V})$ and the positive $(0$ to $2.5 \mathrm{~V})$ potential ranges. Scan rate: $5 \mathrm{mV} / \mathrm{s}$. Bottom: simultaneous dilatation record

Fig. 7. The potential limits were chosen so as to obtain a maximum charge capacity without obvious deterioration over a few cycles. Intercalation and concomitant expansion start at $-1.5 \mathrm{~V}$ and $+2.0 \mathrm{~V}$, respectively. The maximum charge capacities amount to $-146.5 \mathrm{~A} \mathrm{~s} / \mathrm{g}$ with $84.8 \%$ Coulombic efficiency (third cycle) and $138.6 \mathrm{~A} \mathrm{~s} / \mathrm{g}$ with $68.4 \%$ efficiency. The relative height change for the positive potential range is significantly bigger for the graphite electrode than for the 10 times thicker EDLC electrode (Fig. 6). Upon cycling 
in the negative potential range a continuous shrinking of the graphite electrode is observed, making it difficult to estimate the expansion during cation intercalation. For both intercalation/deintercalation processes, a pronounced hysteresis is observed.

\section{4}

\section{Discussion}

We have demonstrated for the first time that a reversible expansion of the electrode material occurs in typical EDLCs utilizing a high surface area activated carbon and an aprotic electrolyte solution. The observed effect is orders of magnitude larger than the one reported for double-layer charging of different carbons in aqueous electrolyte solutions [9-11]. Such pronounced dimensional changes during charge/discharge cycles are generally believed to cause or at least to contribute to degradation and cycle life limitation in batteries. For EDLCs, which have demonstrated a cycle life of several hundred thousand cycles, the observation of dimensional changes of the electrode material is rather surprising. On the other hand, if one could understand this process one might be able to improve the stability of EDLCs even further.

As mentioned in the introduction, we could identify three possible processes leading to charge-induced dimensional changes of carbon materials. Intralayer $\mathrm{C}-\mathrm{C}$ bond length widening, however, can be neglected on the basis of our results. First of all, injection of negative charge is predicted to cause expansion, while injection of positive charge should cause shrinking. In contrast, we observe expansion for either sign of the charge. Moreover, the predicted effect is much smaller than the presently measured one. Consequently, this effect can be excluded.

Likewise, the surface-tension hypothesis [9-11] can only account for small expansion effects $(<0.05 \%)$ that are orders of magnitude smaller than the presently observed ones. Hence, this explanation can also be abandoned.

However, in this context another, somehow related, hypothesis should be mentioned. When the carbon is envisaged as an agglomeration of nanometer-sized crystallites (as it is in the surface-tension hypothesis), the observed expansion might as well be due to the insertion of ions between these particles, and not due to the insertion between the graphene planes of the individual particles. We cannot fully exclude this explanation on the basis of the present results. However, for such an interparticle distance increase, one would expect a decrease of the electronic mobility across the electrode, i.e. between the particles. This is in clear contradiction to previous findings, according to which the electronic conductance of AC electrodes (which is governed by both the mobility and the density of charge carriers) does significantly increase during charging $[17,18]$.

In conclusion, the most probable effect leading to the observed dimensional changes is ion intercalation. For highly graphitized carbons, the electrochemical intercalation of both cations and anions from aprotic solutions is well established. In particular, the intercalation of tetraalkylammonium cations from aprotic solutions has been demonstrated by Besenhard [19,20] and other workers [21,22]. Santhanam and Noel [21] evidenced the intercalation of tetrafluorobo- rate anions into graphite from acetonitrile solution by X-ray diffraction.

Only recently, we observed the intercalation/deintercalation of both anions and cations from $\mathrm{TEABF}_{4} /$ propylencarbonate solution into highly oriented pyrolytic graphite by means of in situ atomic force microscopy [23]. However, such intercalation effects were not discussed previously in the context of activated carbon based double layer capacitors. Our observations also open some analogy to the intercalation of alkali-metal ions $\left(\mathrm{Li}^{+}\right.$and $\left.\mathrm{Na}^{+}\right)$into the nanocrystallites of hard carbons [24].

Recently, JEOL Ltd. (Japan) introduced a novel high energy electrochemical ('Nanogate') capacitor, which utilizes a petroleum coke based $\mathrm{KOH}$ activated carbon with a surface area of about $100 \mathrm{~m}^{2} / \mathrm{g}$, and a solution of TEABF 4 in propylene carbonate [25]. Only upon first charging to above $2.5 \mathrm{~V}$ (cell voltage) is the capacitance developed by ion (and probably also solvent) intercalation into this special carbon [26]. During intercalation a significant increase in pressure is observed for a dimensionally fixed set-up [26]. Takeuchi et al. [27] also measured the onset of insertion processes as a function of graphene layer spacing $(d)$ of the pristine material and found that the onset voltage increases almost linearly from below $2 \mathrm{~V}(d=0.38 \mathrm{~nm})$ to above $3 \mathrm{~V}(d=0.36 \mathrm{~nm})$. This observation may also explain the high onset voltage of about $3.5 \mathrm{~V}$ (Fig. 7) for intercalation in graphite and the significant lower onset for AC (about 1.5 V, Fig. 6).

In order to estimate the contribution of insertion/intercalation processes to the overall charge capacity we calculated the relative expansion for graphite and AC vs. the accumulated charge. Figure 8 shows the relative height changes of the graphite and the EDLC electrodes, respectively, as a function of the mass specific charge. For clarity, only the charging branches of the third cycle (starting at $0 \mathrm{~V}$ ) are shown. The results clearly show that the relative expansion of graphite for a specific charge is larger than the respective expansion of AC. This difference was to be expected in view of the very different surface areas per gram. For AC, about every second or third carbon atom is part of the huge inner surface and

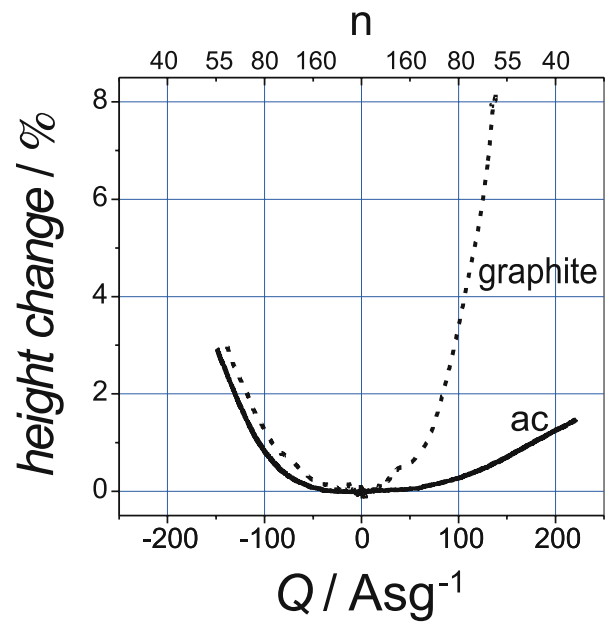

FIGURE 8 Relative height change of the graphite electrode (dashed line) and the EDLC electrode (solid line) upon charging as a function of the mass specific charge. (Charging data of third cycle taken from Figs. 6 and 7.) Also shown is the number of carbon atoms per elementary charge, $n$ 
therefore is accessible to double-layer charging at the ip. In contrast, the graphene layers of graphite (with a negligible surface area per gram) only become accessible upon intercalation; expansion is therefore a pre-requisite for charge storage in graphite.

A quantitative comparison of the height changes of the two electrodes, however, is difficult. First, expansion is not necessarily uniform inside the electrodes (due to different forces acting in the normal and the horizontal directions of the electrode, and due to possible anisotropies inside the electrodes). Second, part of the volume expansion might not result in a height change, but instead in a void volume reduction inside the electrode. Third, irreversible swelling or shrinkage may be superimposed on the recorded height changes. This is obviously the case for the positive branch of AC (slight swelling, Fig. 6) and even more pronounced for the negative branch of graphite (shrinkage, Fig. 7). Therefore, the comparison of both electrode materials shown in Fig. 8 misleads one to strongly under-estimate graphite expansion upon negative charging, and to slightly over-estimate EDLC expansion upon positive charging.

Ignoring the above caveat, and comparing only the positive branches in Fig. 8, we estimate the intercalation contribution to be about $10 \%$ (at $Q=90 \mathrm{~A} \mathrm{~s} / \mathrm{g}$, corresponding to the fully charged capacitor with $U=2.5 \mathrm{~V}$ ).

It can also be seen from Fig. 8 that the charge specific expansion is different for cation and anion insertions. For AC the charge specific expansion (at $Q=90 \mathrm{~A} \mathrm{~s} / \mathrm{g}$ ) is a factor 2.7 higher for cation insertion. For comparison, the size ratio of the unsolvated ions is only 1.5 [28]. This discrepancy might indicate that the contribution of intercalation processes to the overall capacity is more pronounced for the negative EDLC electrode.

For graphite, the opposite trend is observed; the charge specific expansion (at $Q=90 \mathrm{~A} \mathrm{~s} / \mathrm{g}$ ) is now a factor 2.7 higher for anion insertion, suggesting strong co-intercalation of solvent.

One may wonder, then, why the EDLC current characteristics (Figs. 5 and 6) do not show the typical intercalation/deintercalation peaks. In analogy to $\mathrm{Li}$ insertion into nanoporous hard carbons [24], this discrepancy may be explained by the heterogeneity and the poor crystallinity of activated carbon. The correspondingly broad distribution of adsorption/intercalation site energies then results in a pseudocapacitive behavior.

Also, these pseudocapacitive intercalation processes might contribute to the butterfly-like shape of the $\mathrm{CV}$, i.e. the increase of the capacity with increasing excess charge. One should note, however, that the carbon electronic band structure may give a reasonable explanation for this finding [18, 29].

\section{Conclusion}

We have demonstrated for the first time that charging and discharging of a typical electrochemical double layer capacitor based on AC and an aprotic organic electrolyte is connected with a noticeable expansion of the carbon electrode. Expansion can be observed for a capacitor voltage below $2 \mathrm{~V}$. Since dimensional changes are always related to stability and lifetime issues of electrochemical devices, con- trol of this process is important for optimizing the cycle life of EDLCs.

In the context of accelerated life tests, it becomes evident that cycle tests of EDLCs will affect degradation in a different way than constant voltage load tests. While for the former periodic dimensional changes contribute to the degradation, the latter test will be more sensitive to the thermodynamics of the electrode processes.

Finally, we demonstrated that ion insertion into special carbons, which is the fundamental effect utilized in the 'Nanogate' high energy capacitor of JEOL, can also be observed for AC-based EDLCs.

We believe that investigation and control of the ion insertion process will be of fundamental importance for further development and optimization of EDLCs.

ACKNOWLEDGEMENTS Financial support of this work by the Swiss CTI (Project Nos. 5807.2 KTS-NM and 5945.2 TNS) and by Maxwel Technologies SA (Rossens, Switzerland) is gratefully acknowledged. We thank Maxwell Technologies SA (Rossens, Switzerland), Timcal SA (Bodio, Switzerland), and Honeywell Specialties (Seelze, Germany) for providing the EDLC electrode material, the graphite, and the electrolyte solution, respectively.

\section{REFERENCES}

1 M.S. Dresselhaus, G. Dresselhaus, Adv. Phys. 51, 1 (2002)

2 J.R. Dahn, R. Fong, M.J. Spoon, Phys. Rev. B 42, 6424 (1990)

3 L. Pietronero, S. Strässler, Phys. Rev. Lett. 47, 593 (1981)

4 M. Kertesz, Mol. Cryst. Liq. Cryst. 126, 103 (1985)

5 C.T. Chan, W.A. Kamitakahara, K.M. Ho, Phys. Rev. Lett. 58, 1528 (1987)

6 D.E. Nixon, G.S. Parry, J. Phys. C: Solid State Phys. 2, 1732 (1969)

7 W.A. Kamitakahara, J.L. Zarestky, P.C. Eklund, Synth. Met. 12, 301 (1985)

8 J.E. Fischer, H.J. Kim, V.B. Cajipe, Phys. Rev. B 36, 4449 (1987)

9 Y. Oren, I. Glatt, A. Livnat, O. Kafri, A. Soffer, J. Electroanal. Chem. 187, 59 (1985)

10 Y. Oren, A. Soffer, J. Electroanal. Chem. 206, 101 (1986)

11 D. Golub, Y. Oren, A. Soffer, Carbon 25, 109 (1987)

12 W. Biberacher, A. Lerf, J.O. Besenhard, H. Möhwald, T. Butz, Mater. Res. Bull. 17, 1385 (1982)

13 J.O. Besenhard, M. Winter, J. Yang, W. Biberacher, J. Power Source 54, 228 (1995)

14 M. Winter, G.H. Wrodnigg, J.O. Besenhard, W. Biberacher, P. Novak, J. Electrochem. Soc. 147, 2427 (2000)

15 T. Ohzuku, N. Matoba, K. Sawai, J. Power Source 97-98, 73 (2001)

16 DFT User's Guide v1.03, Micromeritics Instrument Corporation (1993)

17 B. Kastening, M. Hahn, B. Rabanus, M. Heins, U. Zum Felde, Electrochim. Acta 42, 2789 (1997)

18 M. Hahn, M. Baertschi, O. Barbieri, J.-C. Sauter, R. Kötz, R. Gallay, Electrochem. Solid-State Lett. A33, 7 (2004)

19 J.O. Besenhard, H.P. Fritz, J. Electroanal. Chem. 53, 329 (1974)

20 J.O. Besenhard, Carbon 14, 111 (1976)

21 R. Santhanam, M. Noel, J. Power Source 66, 47 (1997)

22 C. Dano, J. Simonet, J. Electroanal. Chem. 564, 115 (2004)

23 F.P. Campana, Thesis, University of Bern (2005)

24 D.A. Stevens, J.R. Dahn, J. Electrochem. Soc. 148, A803 (2001)

25 H. Nakamura, M. Okamura, in 13th International Seminar on Double Layer Capacitors and Hybrid Energy Storage Devices, Deerfield Beach, FL (Florida Educational Seminars, Boca Raton, FL, 2003), p. 215

26 M. Takeuchi, K. Koike, T. Maruyama, A. Mogami, M. Okamura, Electrochemistry 66, 1311 (1998)

27 M. Takeuchi, T. Maruyama, K. Koike, A. Mogami, T. Oyama, H. Kobayashi, Electrochemistry 69, 487 (2001)

28 M. Ue, in 8th International Seminar on Double Layer Capacitors and Similar Energy Storage Devices, Deerfield Beach, FL (Florida Educational Seminars, Boca Raton, FL, 1998)

29 H. Gerischer, J. Phys. Chem. 89, 4249 (1985) 